\title{
Systematic errors in temperature estimates from MODIS data covering the western Palearctic and their impact on a parasite development model
}

\author{
Jorge Alonso-Carné ${ }^{1,4}$, Alberto García-Martín ${ }^{2,4}$, Agustin Estrada-Peña ${ }^{3}$ \\ ${ }^{1}$ Department of Geography and Territorial Planning, University of Zaragoza, Zaragoza, Spain; ${ }^{2}$ Centro \\ Universitario de la Defensa de Zaragoza, Academia General Militar, Zaragoza, Spain; ${ }^{3}$ Department of Animal \\ Pathology, Faculty of Veterinary Medicine, University of Zaragoza, Zaragoza, Spain; ${ }^{4}$ GEOFOREST group - \\ Instituto Universitario de Ciencias Ambientales (IUCA), University of Zaragoza, Zaragoza, Spain
}

\begin{abstract}
The modelling of habitat suitability for parasites is a growing area of research due to its association with climate change and ensuing shifts in the distribution of infectious diseases. Such models depend on remote sensing data and require accurate, high-resolution temperature measurements. The temperature is critical for accurate estimation of development rates and potential habitat ranges for a given parasite. The MODIS sensors aboard the Aqua and Terra satellites provide high-resolution temperature data for remote sensing applications. This paper describes comparative analysis of MODISderived temperatures relative to ground records of surface temperature in the western Palaearctic. The results show that MODIS overestimated maximum temperature values and underestimated minimum temperatures by up to $5-6{ }^{\circ} \mathrm{C}$. The combined use of both Aqua and Terra datasets provided the most accurate temperature estimates around latitude $35-44^{\circ} \mathrm{N}$, with an overestimation during spring-summer months and an underestimation in autumn-winter. Errors in temperature estimation were associated with specific ecological regions within the target area as well as technical limitations in the temporal and orbital coverage of the satellites (e.g. sensor limitations and satellite transit times). We estimated error propagation of temperature uncertainties in parasite habitat suitability models by comparing outcomes of published models. Error estimates reached $36 \%$ of annual respective measurements depending on the model used. Our analysis demonstrates the importance of adequate image processing and points out the limitations of MODIS temperature data as inputs into predictive models concerning parasite lifecycles.
\end{abstract}

Keywords: MODIS, temperature, comparative errors, developmental models of parasites.

\section{Introduction}

Several events occurred during the final decades of the $20^{\text {th }}$ Century and the beginning of the $21^{\text {st }}$ Century that indicate global increases in the scope and magnitude of tick-borne infections (Munderloh and Kurtti, 2010). The recognised number of distinct and epidemiologically significant pathogens transmitted by ticks has increased considerably during the last 30 years (Patz et al., 2005). The increase relates to a complex array of factors, including landscape, climate, wildlife hosts and tick distribution, all of which may coalesce to create regional pockets of intensified risk (Rogers and Randolph, 2006). The influence of climate trends exerted on the life cycle of ticks and other parasitic arthropods is an area of increasingly detailed and urgent inquiry. Models are being developed to

Corresponding author:

Augustin Estrada-Peña

Faculty of Veterinary Medicine, University of Zaragoza

Miguel Servet 177, 50013-Zaragoza, Spain

Tel. +34 976761 558; Fax: +34 976761612

E-mail: aestrada@unizar.es account for the full range of climate change scenarios and their impact on tick populations (Ogden et al., 2005).

Predictive habitat distribution modelling is a powerful tool used in both applied and theoretical ecology (Guisan and Zimmermann, 2000; Austin, 2002; Guisan and Thuiller, 2005). These models are used to assess the potential consequences of climate change on the distribution of parasites and vectors of known impact as they relate to animal and human health (Rogers et al., 2002; Ostfeld et al., 2005; Estrada-Peña and Venzal, 2007). Integrating climate data with known physiological tolerances of a given species allows models to address direct and indirect consequences of climate change scenarios on species and ecological systems.

Microclimatic factors impose significant seasonal constraints on tick population dynamics because most of the organism's life span occurs away from the host. Off-host activity includes either actively questing for hosts or transitioning between major life cycle stages. Both of these types of activity are temperature dependent (Randolph, 2004). Process-driven models thus 
address life cycle development throughout stage classifications, each of which depend on temperature. Surface temperature provides the most robust estimates of climate effects on the life cycle of ticks and other arthropods (Ogden et al., 2006).

Temperature data are generally categorised as one of two types of measurement: near surface air temperature (Tair) or land surface temperature (LST) (Jin and Dickinson, 2010). Tair values are measured $1.5 \mathrm{~m}$ above the surface at official weather stations with sensors adequately ventilated and shielded from radiation. LST is a radiometric, temperature measurement based on thermal infrared radiation emitted by the Earth's surface (Wan and Li, 2011) and is thus considered a surface temperature proxy. Satellite-derived LST measurements analyse the canopy temperature in vegetated areas (Mildrexler et al., 2011). Models use these inputs to estimate development rates and climate impacts on the potential spread of ticks and pathogens they may transmit (Hancock et al., 2011). These types of study explicitly assume that remote sensing temperature data provide accurate mean temperature estimates for a given daily, biweekly or monthly interval. Remotely sensed temperature data, however, are temperature proxies and do not always reflect the actual conditions for a given location and time interval.

Satellite-derived temperature estimates are also used as variables in modelling the realised climate niche of an arthropod at varying temporal and spatial resolutions (Hay et al., 1997, 2006; Rogers et al., 2002; Rogers and Randolph, 2006). Realised climate niche is the unique set of environmental factors with which the arthropod is associated. These environmental factors are homogeneously distributed within the broader environmental niche as defining the distribution of a particular species. Realised climate niche information is increasingly used to predict the habitat of an arthropod and project its potential geographical and temporal ranges. The spatial nature of these models requires both LST and normalized difference vegetation index (NDVI) inputs derived either from principal component analysis (PCA) or Fourier analysis (Estrada-Peña, 1999; Rogers et al., 2002).

MODIS datasets are well-suited for epidemiological applications due to (i) their enhanced spectral resolution relative to the Advanced Very High Resolution Radiometer (AVHRR), which includes 36 spectral channels with smaller wavelength ranges and significantly improved signal-to-noise ratios (Justice et al., 2002); (ii) a repeat time of one to two days, which gives it a better temporal resolution at a significantly higher spatial resolution relative to AVHRR
(Townshend and Justice, 2002); and (iii) greater accessibility to high quality, fully processed data covering large areas at recent time scales. However, satellite imagery imposes certain constraints on reconciling temperatures and accurately estimating model variables (Liang et al., 2006) and MODIS images and temperature data are accompanied by a set of flags documenting errors in data recording and processing. These flags include filters for snow and/or cloud cover. For models in which temperature plays a primary role, LST data may introduce uncertainty associated with the original measurement or processing error. These intrinsic errors can propagate through process-driven models as uncertainties in direct inputs, through Fourier transform image processing steps (Scharlemann et al., 2008), or during PCA decomposition of uncorrelated factors, which is a method increasingly used in determining habitat for arthropod vectors. Ecological epidemiology and other applications naturally seek to minimize errors in the raw data and identify any operations that may propagate error unnecessarily.

This study systematically compared LST and Tair measurements from a global network of weather stations in the western Palaearctic. It specifically seeks to evaluate the differences between long-term daily temperature data from ground recording stations and LST values as measured, processed and scientifically validated by the MODIS team. The study offers a comparative overview of long-term MODIS data series and specifies caveats pertaining to the use of satellitederived data in epidemiological studies concerning arthropods and arthropod-transmitted pathogens. The approach is unique in its focus on 8-day cycles including both day and night LST values making use of Tair data spanning the period from 2000-2010.

\section{Material and methods}

We focused on the western Palaearctic, a region located between $18^{\circ} \mathrm{W}, 42^{\circ} \mathrm{E}, 26^{\circ} \mathrm{N}$ and $66^{\circ} \mathrm{N}$. According to geopolitical frameworks, this area includes every country west of the border between Russia and Europe, north of the Sahara desert, and east of the Atlantic Ocean. The area is densely populated and at particular risk for the spread of tick-borne pathogens (Estrada-Peña and Venzal, 2007).

The MOD11C2 and MYD11C2 (version 5) datasets of the MODIS series were downloaded for a period spanning the years 2000 to 2010 (https://lpdaac.usgs.gov/products/modis_products_tab le). The datasets include LST measurements from both 
the Terra and Aqua satellites. Each satellite trace a unique orbital path over the study region, resulting in slightly divergent estimates of daily averaged temperature as they apply to ecological processes. The spatial resolution of the dataset is a nominal $0.05^{\circ}$ and it has been developed to "Validated Stage 2", which means that its accuracy has been assessed over a wide-ranging set of locations for different time periods using several ground-truth and validation techniques. For each 8-day period, we used both day and night data from the Terra and Aqua sources. Images were cropped according to the study region's geographic area and temperature measurements were converted to degrees Celsius. Quality flags were addressed by removing pixels that were catalogued by MODIS as being obscured by clouds, water or null/non-valid measurements. For every 8-day interval of the 2000-2010 study period, LST values were integrated with a metric classifying their associated pixels. Parameters affecting the quality of pixels rendered the metric $\mathrm{QC}$ as $1=$ perfect; 2 = optimal; and 3 = valid but moderately affected by water vapour.

The accuracy of the LST data was evaluated by comparing it with a series of temperature measurements collected by ground climate recording stations. The European Climate Assessment and Dataset (ECA\&D) provided daily quality-controlled temperature data for the 2000-2010 study period (available at http:// eca.knmi.nl/). This dataset contained 26,141 observations from 4,824 meteorological stations throughout
Europe and the Mediterranean. Daily station series were collected by the climatological divisions of national meteorological and hydrological services as well as observatories and research centres throughout Europe and the Mediterranean area. Fig. 1 shows the geographic range of the study region, as well as the locations of stations providing data for the study. We referenced and compared MODIS data with the corresponding ECA\&D series. For every 8-day interval of the 2000-2010 study period, the validated average, maximum and minimum temperature values recorded by the ECA\&D series (Tair) were cross-referenced to the validated MODIS values.

A preliminary analysis of the two arrays revealed compelling, first-order, spatial differences. Raw differences between the two arrays were then binned into discrete latitude and longitude units for each 8-day interval to determine whether differences arose from temporal and/or spatial variation. We mapped the study region onto a grid having hexagonal units of $1^{\circ}$ radius and containing errors averaged over each 8 -day interval for the entire 2000-2010 study period. Differences between the data arrays were further analysed according to categorical classifications of ecosystem type for each unit of the grid. Spatial data for ecosystem classification was obtained from http://www.worldwildlife.org/science/data/item1875 .html (accessed on September, 2010). The ecological data was cropped according to the geographic range of the study area and used to designate predominant

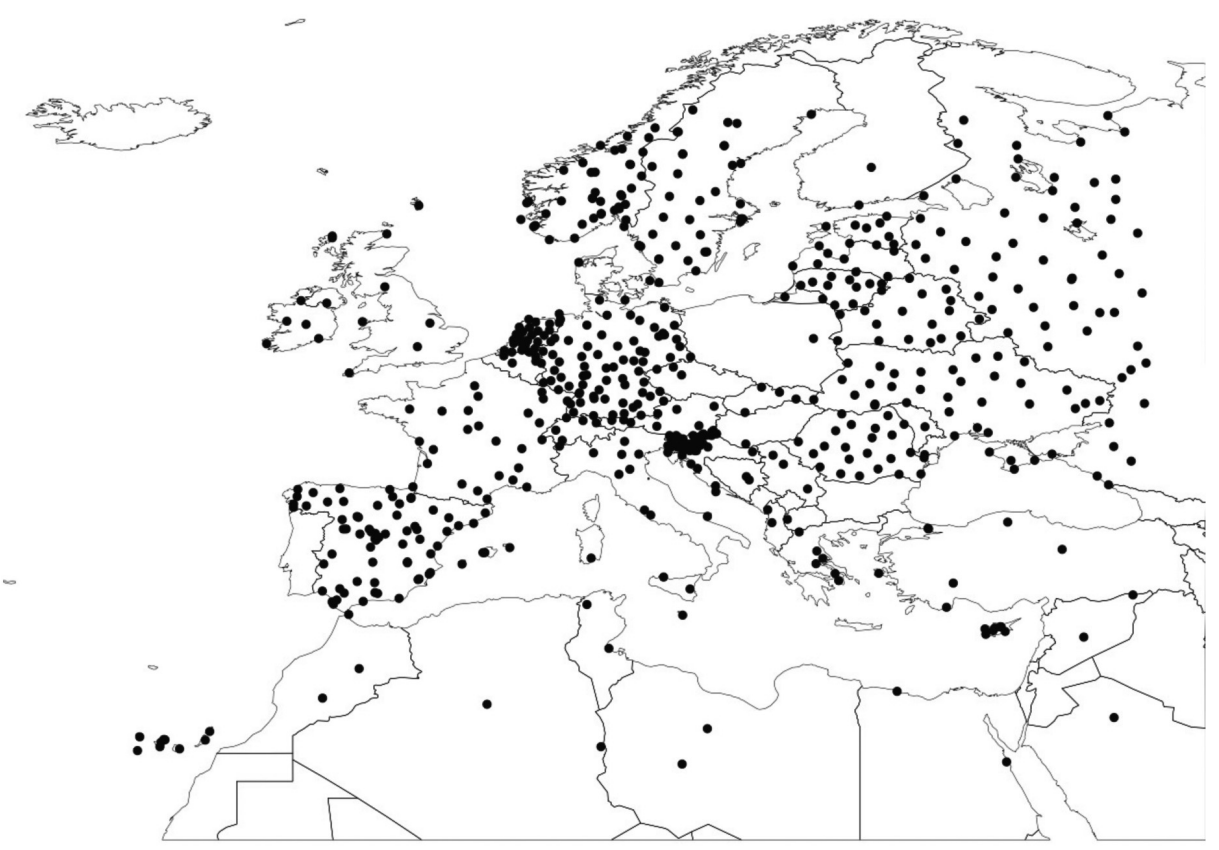

Fig. 1. Geographic distribution of the study area and the ECA\&D stations used for comparison with the temperature data derived from MODIS. 
biomes present in a given region according to established climate and ecological criteria. Besides uncertainties in latitude and longitude, observed errors might arise from differences in the transit time and orbital path of satellites. We therefore cross-tabulated the orbital transit times for each satellite either during the day or the night at a given location, along with the error associated with each measurement and averaged over the entire 2000-2010 study period.

To evaluate uncertainties introduced by MODIS temperature estimates in simple average temperature models, we used a quadratic equation describing the development of the tick Ixodes ricinus from egg to larvae, as reported in Dobson et al. (2011).

$$
Y=-0.00001127 T^{2}+0.002305 T-0.0185
$$

This well-tested equation, where $T$ is the temperature, describes the life cycle of a tick native and common to the study region. It provides a straightforward method to evaluate error propagation and its potential spatial variability.

\section{Results}

Comparison of MODIS and ECA\&D temperature data

Table 1 shows the mean difference between day and night temperatures from the Terra or Aqua satellites and that from ECA\&D ground recording stations. The table includes mean, maximum and minimum temperature values derived from the satellite sensor and measured on the ground. Both the mean maxima of the Terra and Aqua day temperatures and the mean minima of the satellites' night temperatures give minimum errors. MODIS LST values are relatively well correlated with temperatures from ground recording stations as catalogued in the ECA\&D data. Aqua data show better correlation with Tair for day temperatures, whereas Terra data show better correlation with Tair for night temperatures. Averaged errors recorded by Terra ranged from $3-5{ }^{\circ} \mathrm{C}$, while Aqua errors ranged from $5-6{ }^{\circ} \mathrm{C}$ for day and night periods. MODIS tended to overestimate day temperatures and underes-

Table 1. Mean results comparing MODIS LST estimates and ECA\&D temperatures. The mean values include both Terra and Aqua estimates and corresponding ECA\&D observations from ground recording stations).

\begin{tabular}{|c|c|c|c|c|}
\hline \multirow[t]{2}{*}{ Variable } & \multicolumn{2}{|r|}{$\mathrm{QCD}>0$} & \multicolumn{2}{|r|}{$\mathrm{QCD}=1$} \\
\hline & $\mathrm{N}$ & $\begin{array}{c}\text { Mean error } \\
\left({ }^{\circ} \mathrm{C}\right) \pm \text { standard error }(\mathrm{SE})\end{array}$ & $\mathrm{N}$ & $\begin{array}{c}\text { Mean error } \\
\left({ }^{\circ} \mathrm{C}\right) \pm \text { standard error }(\mathrm{SE})\end{array}$ \\
\hline Average of means minus LSTD - Terra & 121,328 & $-3.60 \pm 0.02$ & 81,847 & $-4.92 \pm 0.02$ \\
\hline Average of means minus LSTN- Terra & 127,030 & $5.36 \pm 0.01$ & 61,759 & $5.11 \pm 0.01$ \\
\hline Average of maximum minus LSTD - Terra & 154,033 & $0.93 \pm 0.01$ & 103,343 & $0.04 \pm 0.01$ \\
\hline Average of maximum minus LSTN - Terra & 152,376 & $10.04 \pm 0.01$ & 76,532 & $10.14 \pm 0.01$ \\
\hline Average of minimum minus LSTD - Terra & 133,700 & $-9.18 \pm 0.02$ & 94,876 & $-9.91 \pm 0.02$ \\
\hline Average of minimum minus LSTN - Terra & 141,635 & $0.72 \pm 0.01$ & 69,965 & $0.15 \pm 0.01$ \\
\hline Average of means minus LSTD - Aqua & 83,060 & $-5.14 \pm 0.02$ & 29,452 & $-5.07 \pm 0.03$ \\
\hline Average of means minus LSTN - Aqua & 78,963 & $6.41 \pm 0.01$ & 42,076 & $6.50 \pm 0.01$ \\
\hline Average of maximum minus LSTD - Aqua & 112,127 & $-0.51 \pm 0.01$ & 39,045 & $-0.49 \pm 0.03$ \\
\hline Average of maximum minus LSTN - Aqua & 105,991 & $11.09 \pm 0.01$ & 56,154 & $11.53 \pm 0.02$ \\
\hline Average of minimum minus LSTD - Aqua & 104,516 & $-9.72 \pm 0.02$ & 37,023 & $-9.41 \pm 0.03$ \\
\hline Average of minimum minus LSTN - Aqua & 98,667 & $1.74 \pm 0.01$ & 52,202 & $1.47 \pm 0.01$ \\
\hline
\end{tabular}

$\mathrm{N}$ is the number of 8-day intervals for which data were available for 2000-2010; LSTD, land surface temperature day; LSTN, land surface temperature night; QCD, standard quality flag for pixels in MODIS datasets. The QCD=1 dataset includes only the maximum quality pixels designated by the MODIS team. The QCD $>0$ dataset includes all the pixels with adequate temperature observations, even if observations are obscured by snow, water or ice. 
timate night temperatures. These errors were not strongly impacted by exclusion of data flagged for quality reasons within the MODIS dataset. The average error using only the clear pixels was around $0.2{ }^{\circ} \mathrm{C}$ less than the error term for data that included obscured pixels. The error between ECA\&D mean maximum Tair and MODIS Terra LST values, averaged for the complete 2000-2010 study period, was only $0.04{ }^{\circ} \mathrm{C}$ excluding MODIS quality flags, but reached $0.93{ }^{\circ} \mathrm{C}$ when the flagged pixels were included. Both Tair maximum daily temperature and LST were well correlated over the study period and across all localities, assuming balanced errors in the results.

\section{Geographical analysis of Terra and ECA\&D tempera- tures}

Fig. 2 shows the geographical distribution of errors in maximum temperature observations derived from Terra according to latitude and longitude and integrated over 8-day intervals for the entire 2000-2010 study period. This plot demonstrates that southern latitudes (approximately covering the Mediterranean region) experience the greatest overestimation of
MODIS temperatures. This effect tends to diminish at northern latitudes. Errors were the highest at western and eastern longitudinal extremes of the study area. Thus, while the average of error between maximum Tair and MODIS LST was balanced in the complete dataset (as seen in Table 1), regional analysis showed that MODIS Terra tended to overestimate the temperature for regions of the Palaearctic at low latitudes and western longitudes by up to $5-10{ }^{\circ} \mathrm{C}$ during he summer months. Fig. 2 also shows the error estimates between minimum Tair and MODIS-Terra according to time of year and latitude and longitude gradients. The temperature records derived from MODIS tended to overestimate minimum temperatures at every latitude and longitude with errors being larger during the summer across all geographic locations.

\section{Geographical analysis of Aqua and ECA\&D tempera- tures}

Fig. 3 shows the geographical distribution of errors with respect to maximum temperature observations derived from Aqua according to latitude and longitude and integrated over 8-day intervals for the 2000-2010
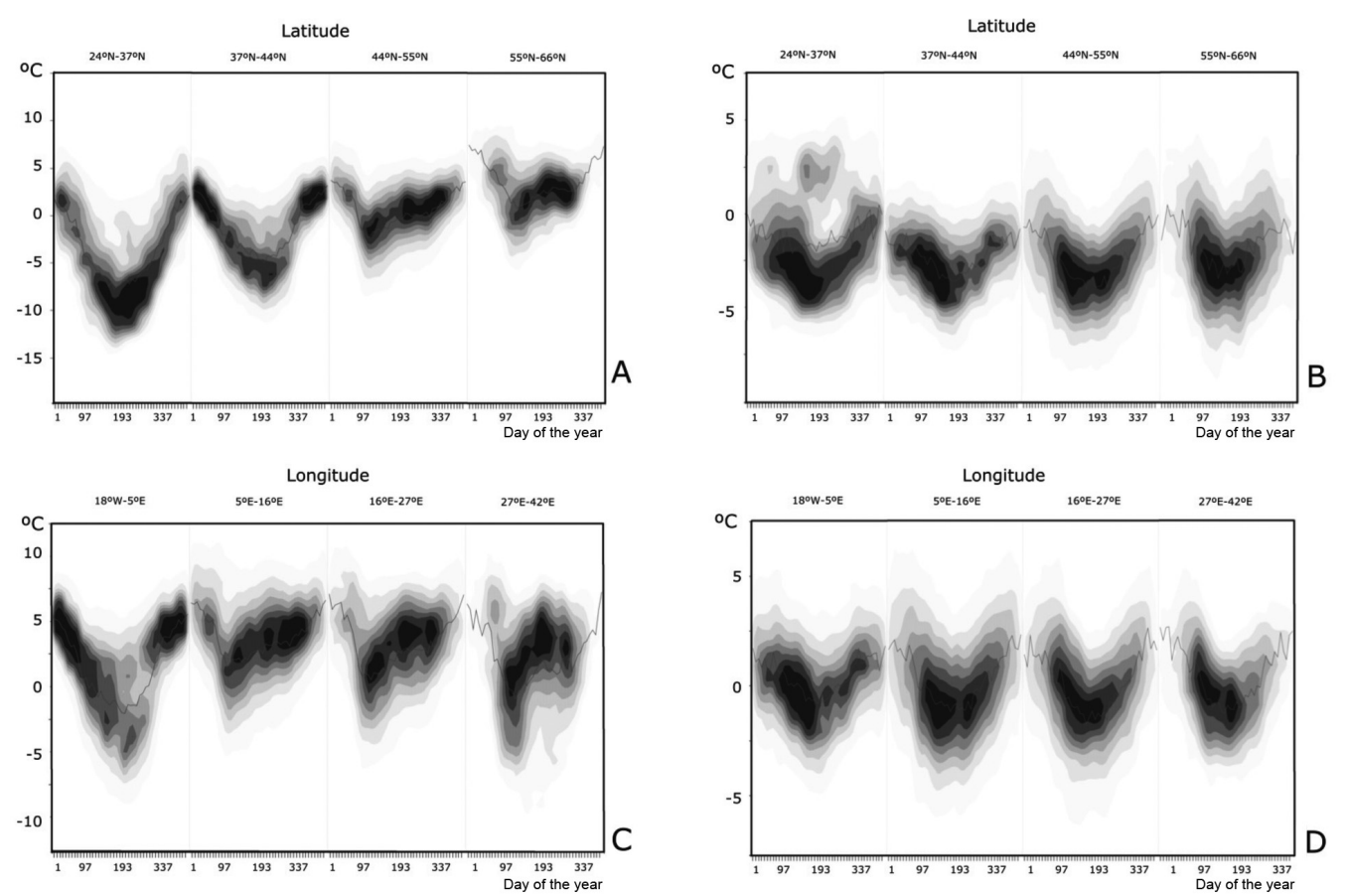

Fig. 2. Plot of the mean temperature error $\left({ }^{\circ} \mathrm{C}\right)$ between MODIS-Terra estimates (either day or night) versus mean observations from ECA\&D ground recording stations, plotted according to latitude and longitude for each day of the study period (the grey scale is proportional to the number of ground stations providing observations. Negative values in each panel represent ground temperature overestimation by MODIS, whereas positive values represent underestimation). Panel A shows differences between average maximum temperatures (ECA\&D) and MODIS Terra LST day versus latitude; Panel B shows differences between average minimum temperatures (ECA\&D) and MODIS Terra LST night versus latitude; Panel C shows differences between average maximum temperatures $(\mathrm{ECA} \& \mathrm{D})$ and MODIS Terra LST day versus longitude; Panel D shows differences between average minimum temperatures (ECA\&D) and MODIS Terra LST night versus longitude. 

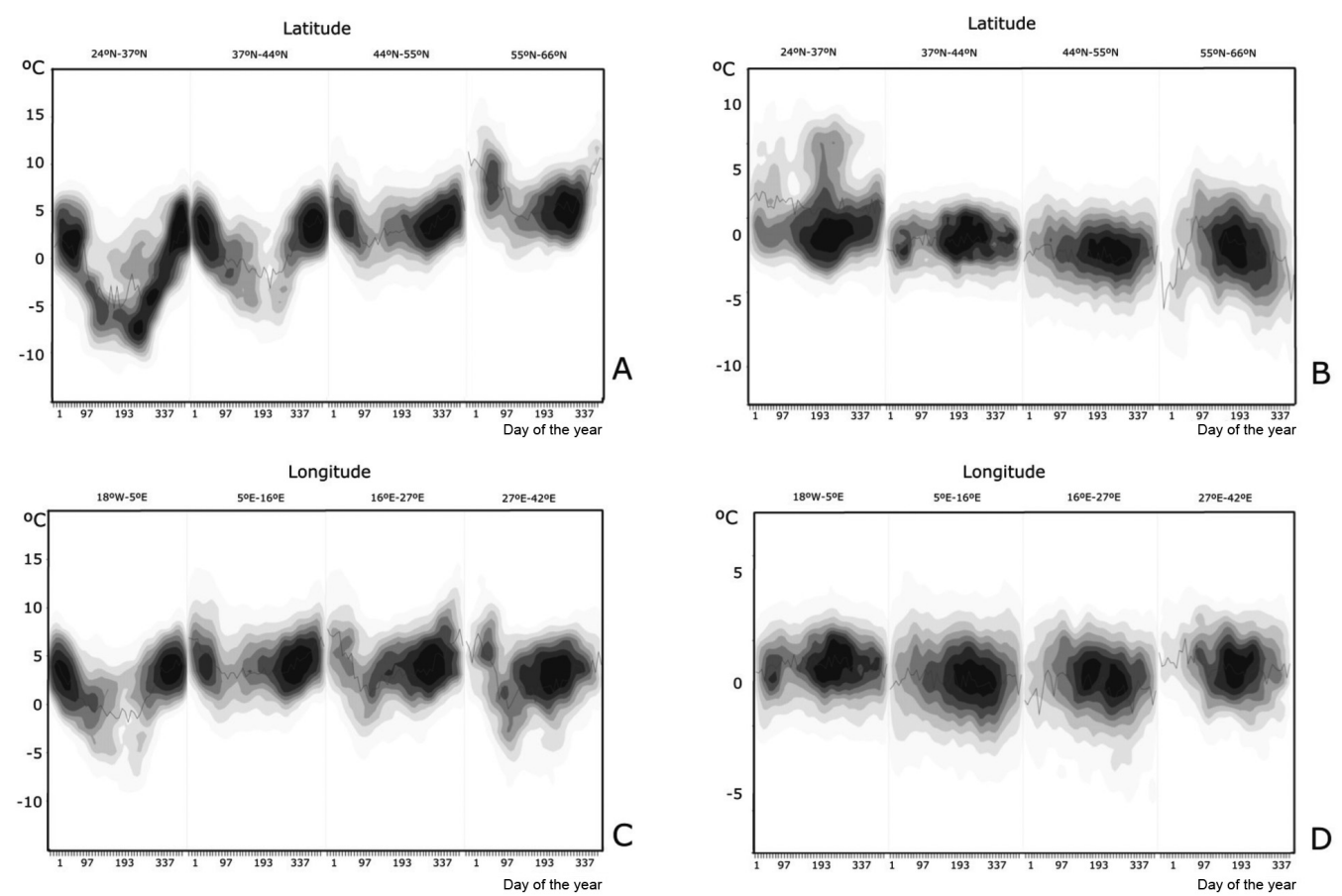

Fig. 3. Plot of mean temperature errors $\left({ }^{\circ} \mathrm{C}\right.$ ) between MODIS-Aqua estimates (day or night) against the mean values from ECA\&D ground recording stations versus latitude and longitude for each day of the study period (the grey scale is proportional to the number of ground stations providing observations. Negative values in each panel represent ground temperature overestimation by MODIS, whereas positive values represent underestimation). Panel A shows differences between average ECA\&D maximum temperatures and MODIS Aqua LST day versus latitude; Panel B shows differences between average ECA\&D minimum temperatures and MODIS Aqua LST night versus latitude; Panel C shows differences between average maximum ECA\&D temperatures and MODIS Terra LST day versus longitude; Panel D shows differences between average minimum ECA\&D temperatures and MODIS Terra LST night versus longitude.

study period. The Aqua data were better correlated with Tair than Terra, especially around latitudes of $40^{\circ}$ $\mathrm{N}$ but with a small overestimation of the night temperatures. Comparison of values according to longitude showed no specific differences and a similar level of error. In order to understand the balance of errors among maximum and minimum temperature observations, we combined both Terra and Aqua day and night values into a single dataset and compared it to similarly formatted data from ground recording stations. Fig. 4 shows the associated errors plotted according to latitude and longitude. They ranged between $+5{ }^{\circ} \mathrm{C}$ (underestimation) and $-10{ }^{\circ} \mathrm{C}$ (overestimation). The largest errors appeared during the summer at low latitudes between latitudes $24^{\circ} \mathrm{N}$ and $44^{\circ} \mathrm{N}$. In the autumn and winter months, MODIS measurements tended to slightly underestimate actual temperatures relative to ground-based recording stations.
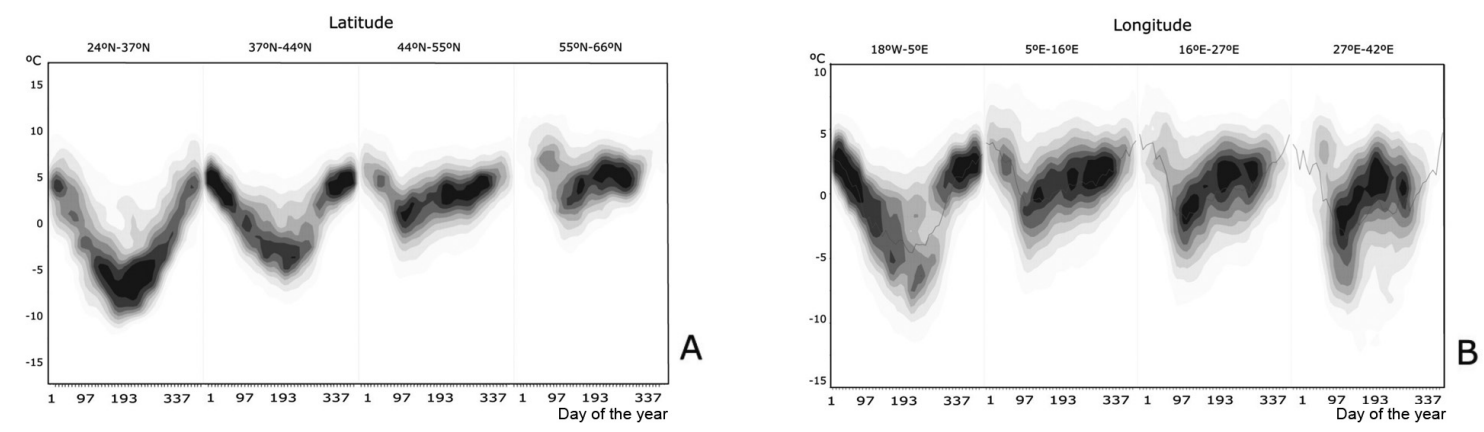

Fig. 4. Mean error between combined maximum and minimum ECA\&D temperatures and combined day and night MODIS Terra and Aqua temperature for each day of the study period, versus latitude (A) and versus longitude (B). 
Spatial relationships of temperature measurement errors

We mapped average errors onto the hexagonal grid of $1^{\circ}$ radius units (Fig. 5). Each cell displays errors averaged over the 2000-2010 study period. The map shows that areas of the Mediterranean basin exhibit the largest overestimation of temperature encountered, while central and northern Europe show large patches of temperature underestimation. Interpretation suggests that this pattern may relate to the predominant biome represented at the surface or the orbital transit time of the satellites, which may or may not coincide with the daily timing of maximum or minimum Tair. To investigate this potential inconsistency, we tabulated minimum and maximum temperatures from either day or night transits for both satellites. These data were compared with estimates for the correlation between temperature error and the primary vegetal ecosystems in a given area as well as estimates between temperature error and orbital transit time. Table 2 lists the errors correlated with major ecosystems of different regions and shows that the largest errors were associated with ecosystems that experience thermal extremes. The largest temperature overestimations correlated with Mediterranean type biomes and esclerophilous plant formations, i.e. poorly developed vegetation, typical of arid environments. It corresponds to the final stages before the desert conditions. The most pronounced underestimations occurred in the vegetal biomes of central and northern Europe such as the Scandinavian and Atlantic mixed or humid forests. Tables 3, 4, 5 and 6 show the average errors calculated relative to the orbital transit time for each satellite along both the ascending and the descending orbital paths. The largest temperature errors were found to be associated with the nocturnal transits. The errors associated with day transits were generally smaller for both satellites. Orbital transits approaching the hour of sunrise, expected to exhibit minimum daily LST and errors for this time-frame, were small relative to that of other periods.

\section{Evaluation of error propagation in parasite develop- ment models}

To estimate how temperature errors may affect analytical models, we entered both ECA\&D and MODIS temperature data into a simple quadratic equation describing development of the tick I. ricinus. The average error for the entire dataset was initially relatively low, well below $2 \%$ for any given 10 -day period. On a regional scale, however, estimated development rates were strongly affected by error in temperature terms. Fig. 6 shows the percentage error in development estimates. Temperature uncertainties would cause the most significant overestimation of tick development rates in the Mediterranean basin (between $30^{\circ} \mathrm{N}$ to $45^{\circ} \mathrm{N}$ latitude) with an average maximum overestimation of $2-4 \%$ per 10 -day interval during the summer. The uncertainties would further cause a $1 \%$ underesti-

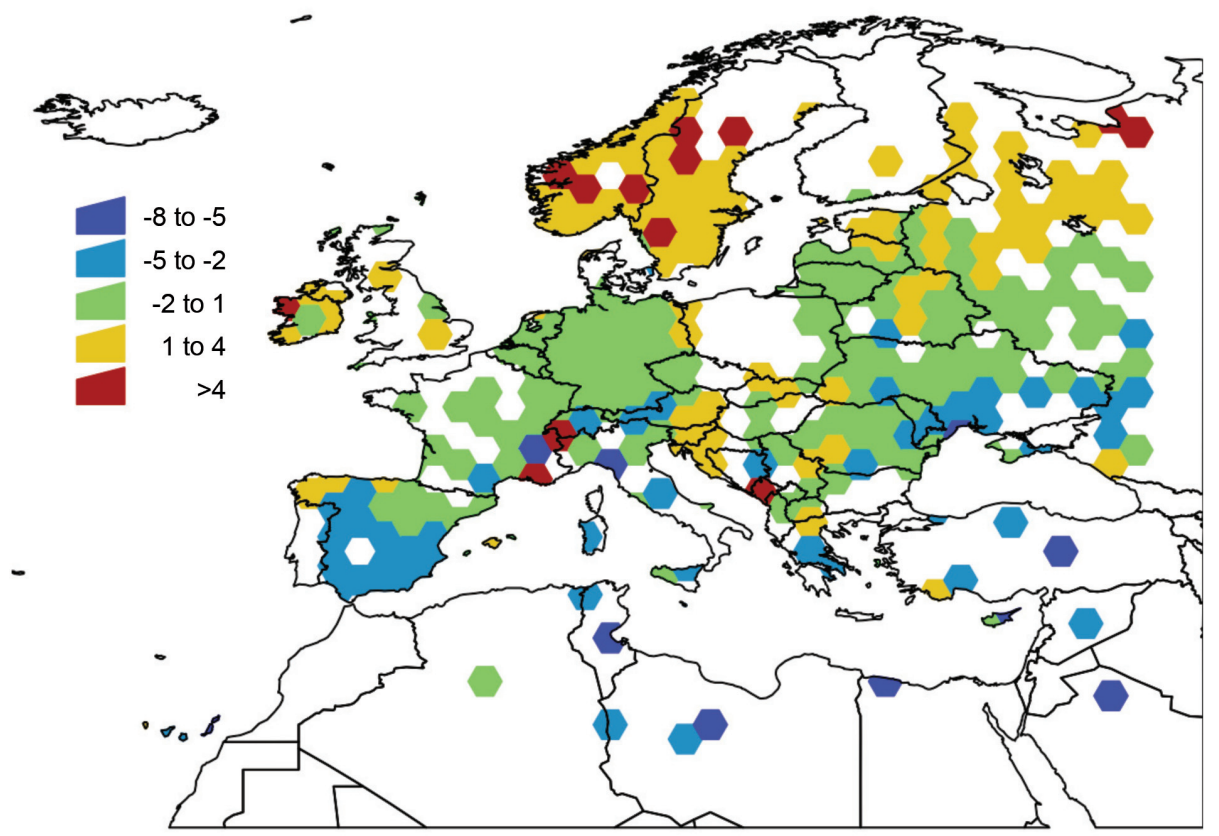

Fig. 5. Europe map of errors between ECA\&D and MODIS temperature measurements (data are shown at a polygonal resolution with hexagons of a $1^{\circ}$ radius, which are coloured according to the average value of error terms as calculated from all stations within the cell. Areas without colour-code had no hexagons with validated ECA\&D data, and thus could not provide temperature error estimates). 

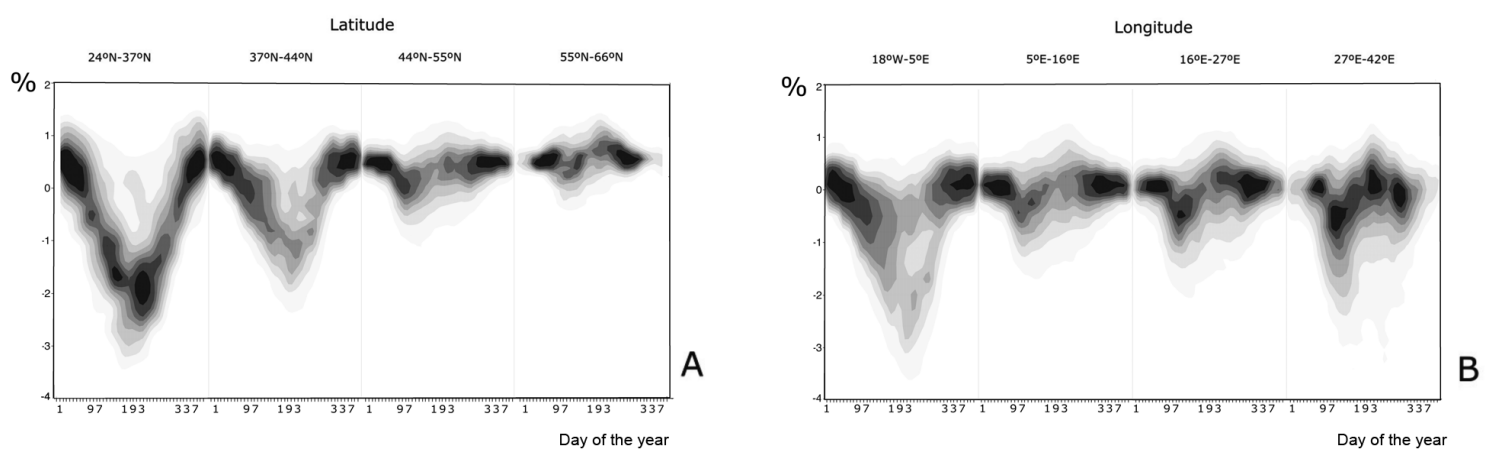

Fig. 6. Mean error for the development of eggs belonging to the tick Ixodes ricinus using an equation described in Dobson et al. (2011) versus latitude (A) and longitude (B) for each day of the study period (error terms reflect differences between developmental equation results based on ECA\&D temperature input and that based on MODIS input for the same geographic location. The grey scale is proportional to the number of ground stations providing observations).

Table 2. Mean errors derived from comparisons of MODIS LST measurements and ECA\&D temperatures (ascending list with the error greater than $1^{\circ} \mathrm{C}$. Error terms include data from both Terra and Aqua satellites relative to ECA\&D ground recording station temperature data. Negative values reflect underestimation by MODIS).

Type of ecological region

Mean temperature error

$\left({ }^{\circ} \mathrm{C}\right) \pm$ standard error $(\mathrm{SE})$

Po Basin mixed forests

$-1.23 \pm 0.11$

Aegean and Western Turkey sclerophyllous and mixed forests

$-1.60 \pm 0.11$

Pontic steppe

$-1.82 \pm 0.07$

Iberian conifer forests

$-1.92 \pm 0.12$

Crimean sub-Mediterranean forest complex

$-2.05 \pm 0.41$

Tyrrhenian-Adriatic Sclerophyllous and mixed forests

$-2.06 \pm 0.20$

Northern Anatolian conifer and deciduous forests

$-2.18 \pm 0.20$

Canary Islands dry woodlands and forests

$-2.55 \pm 0.18$

Southwest Iberian Mediterranean sclerophyllous and mixed forests

$-2.81 \pm 0.10$

Iberian sclerophyllous and semi-deciduous forests

$-2.98 \pm 0.04$

Anatolian conifer and deciduous mixed forests

$-3.06 \pm 0.20$

South-eastern Iberian shrubs and woodlands

$-4.17 \pm 0.22$

Cyprus Mediterranean forests

$-5.09 \pm 0.16$

Central Anatolian steppe and woodlands

$-5.58 \pm 0.52$

Mediterranean acacia-argania, dry woodlands and succulents

$-6.16 \pm 0.23$

North Saharan steppe and woodlands

$-6.38 \pm 0.20$

Red Sea Nubo-Sindian tropical desert and semi-desert

$-7.18 \pm 0.31$

Apennine deciduous mountain forests

$-7.46 \pm 0.25$

Mediterranean dry woodlands and steppe

$-8.45 \pm 0.37$

Pannonian mixed forests

$1.04 \pm 0.03$

Cantabrian mixed forests

$1.30 \pm 0.06$

Illyrian deciduous forests

$1.30 \pm 0.08$

Carpathian mountain forests

$1.56 \pm 0.13$

Sarmatic mixed forests

$1.68 \pm 0.04$

North Atlantic moist mixed forests

$1.71 \pm 0.15$

Celtic broadleaf forests

$1.85 \pm 0.10$

Scandinavian coastal conifer forests

$2.17 \pm 0.09$

Scandinavian and Russian taiga

$3.76 \pm 0.03$

Scandinavian mountain birch forests and grasslands

$4.14 \pm 0.15$ 
Table 3. Tabulation of Terra satellite transit during daytime and the error resulting from comparing temperatures registered to the maximum temperatures observed by ECA\&D ground recording stations (the MODIS derived data included only highquality pixels).

\begin{tabular}{lrc}
\hline $\mathrm{GMT}^{\mathrm{a}}$ & $\mathrm{N}^{\mathrm{b}}$ & $\begin{array}{c}\text { Mean temperature } \\
\text { error }\left({ }^{\circ} \mathrm{C}\right) \pm \mathrm{SE}^{\mathrm{c}}\end{array}$ \\
\hline $7.30-8.30$ & 456 & $3.00 \pm 1.03$ \\
$8.30-9.30$ & 5,980 & $2.63 \pm 0.11$ \\
$9.30-10.30$ & 32,350 & $4.67 \pm 0.06$ \\
$10.30-11.30$ & 51,612 & $4.07 \pm 0.04$ \\
$11.30-12.30$ & 12,712 & $3.39 \pm 0.15$ \\
$12.30-13.30$ & 173 & $5.04 \pm 0.69$
\end{tabular}

${ }^{\mathrm{a}}$ Greenwich Mean Time; ${ }^{\mathrm{b}}$ number of 8-day intervals for which 2000-2010 data was available; 'standard error.

Table 4. Tabulation of Aqua satellite transit during the day and the errors resulting from comparing temperatures registered to the maximum temperatures observed by ECA\&D ground recording stations (the MODIS derived data included only highquality pixels).

\begin{tabular}{lrc}
\hline $\mathrm{GMT}^{\mathrm{a}}$ & $\mathrm{N}^{\mathrm{b}}$ & $\begin{array}{c}\text { Mean temperature } \\
\text { error }\left({ }^{\circ} \mathrm{C}\right) \pm \mathrm{SE}^{\mathrm{c}}\end{array}$ \\
\hline $8.00-9.30$ & 491 & $6.63 \pm 0.92$ \\
$9.30-11.00$ & 4,698 & $4.01 \pm 0.20$ \\
$11.00-12.30$ & 18,487 & $4.39 \pm 0.07$ \\
$12.30-14.00$ & 12,503 & $2.54 \pm 0.08$ \\
$14.00-15.30$ & 3,200 & $0.82 \pm 0.59$ \\
\hline
\end{tabular}

${ }^{\mathrm{a}}$ Greenwich Mean Time; ${ }^{\mathrm{b}}$ number of 8-day intervals for which 2000-2010 data was available; 'standard error.

mation per 10-day interval in development rates at northern latitudes in autumn and winter. This underestimation should be obvious at latitudes of up to $44^{\circ}$ $\mathrm{N}$ for most periods of the year reaching latitudes of $55^{\circ}$ $\mathrm{N}$ for the entire annual dataset. With respect to longitude, MODIS records overestimated development at every longitude with maximum overestimates during the summer in the West, and during late spring in the East. Underestimation of development rates never exceeded values of $1 \%$ per 10 -day interval for some regions of the longitudinal gradient studied.

\section{Discussion}

This paper analysed a point-scale, data assimilation scheme based on recording station temperature data and satellite-derived temperature estimates. It primarily aimed to quantify errors in MODIS estimates of LST. Error propagation in parasite development models that seek to estimate tick life cycles was also addressed. A
Table 5. Tabulation of Terra satellite transit during night and the errors resulting from comparing temperatures registered to the minimum temperatures observed by ECA\&D ground recording stations (the MODIS derived data included only highquality pixels).

\begin{tabular}{lrc}
\hline $\mathrm{GMT}^{\mathrm{a}}$ & $\mathrm{N}^{\mathrm{b}}$ & $\begin{array}{c}\text { Mean temperature } \\
\text { error }\left({ }^{\circ} \mathrm{C}\right) \pm \mathrm{SE}^{\mathrm{c}}\end{array}$ \\
\hline $18.30-19.30$ & 1,516 & $-3.78 \pm 0.37$ \\
$19.30-20.30$ & 13,081 & $-3.67 \pm 0.07$ \\
$20.30-21.30$ & 32,559 & $-3.63 \pm 0.04$ \\
$21.30-22.30$ & 18,467 & $-2.90 \pm 0.05$ \\
$22.30-23.30$ & 3,675 & $-0.47 \pm 0.16$ \\
$23.30-0.30$ & 106 & $0.97 \pm 1.04$ \\
\hline
\end{tabular}

${ }^{\mathrm{a}}$ Greenwich Mean Time; ${ }^{\mathrm{b}}$ number of 8 -day intervals for which 2000-2010 data was available; 'standard error.

Table 6. Tabulation of Aqua satellite transit during night-time and the error resulting from comparing temperatures registered to the minimum temperatures observed by ECA\&D ground recording stations (the MODIS derived data included only highquality pixels).

\begin{tabular}{lrc}
\hline $\mathrm{GMT}^{\mathrm{a}}$ & $\mathrm{N}^{\mathrm{b}}$ & $\begin{array}{c}\text { Mean temperature } \\
\text { error }\left({ }^{\circ} \mathrm{C}\right) \pm \mathrm{SE}^{\mathrm{c}}\end{array}$ \\
\hline $0.00-1.00$ & 11,398 & $-2.67 \pm 0.18$ \\
$1.00-2.00$ & 14,608 & $-2.25 \pm 0.06$ \\
$2.00-3.00$ & 5,255 & $-0.46 \pm 0.14$ \\
$3.00-4.00$ & 333 & $-0.83 \pm 0.59$ \\
\hline
\end{tabular}

${ }^{a}$ Greenwich Mean Time; ${ }^{b}$ number of 8-day intervals for which 2000-2010 data was available; 'standard error.

better understanding of these effects can minimize error and error propagation using image quality filters and other operations. Vector-borne diseases are highly sensitive to changes in climate due to the effects of environmental conditions on a given vector or pathogen's development rate (Guis et al., 2012).

Health risks associated with climate change call for mapping of potential climate impacts on the distribution and population dynamics of health-threatening arthropods (Patz et al., 2005; Estrada-Peña et al., 2012). Such efforts commonly use interpolated climate data from sources such as the increasingly popular Worldclim (Hijmans et al., 2005). The availability of remote sensing data has enhanced its use in modelling of insect-related health risks. Many authors have thoroughly reviewed MODIS imagery and pointed out its usefulness for ecological studies in vector mapping (Brooker et al., 2001; Rogers et al., 2002; Hay et al., 2006). Tatem et al. (2006) previous studies demonstrated the importance of processing the raw data files with filters provided by the 
MODIS team to remove noise coming from water contamination (Scharlemann et al., 2008), which biases LST estimates. However, apart from adequate filtering, studies using MODIS data should also develop an a priori understanding of the potential divergence between LST and Tair values considering how it may affect the model of interest.

Ground temperature is a key variable in estimating tick development (Randolph, 2004), especially cohorts of ticks recruited into questing upon completion of the preceding molting period, which is due to temperature effects that regulate this and other activities (Randolph et al., 2002). Prediction of the relative roles of these factors plays a critical role in life cycle modelling as does anticipating the spread of the ticks (Danielova et al., 2006; Jaenson and Lindgren, 2011) and monitoring the pathogens they transmit (Jaenson et al., 2012). Divergent temperature estimates between ground recording stations (or laboratory studies on development) and remote sensing instruments can introduce systematic errors that obscure otherwise regionally coherent findings regarding this issue.

We observed a strong positive correlation between LST measured during nocturnal transit and minimum Tair. Maximum LST increases faster than maximum Tair with rising ambient temperatures and thus captures additional information on the concentration of thermal energy within a given area of the Earth's surface. Maximum Tair therefore exhibits lesser correlation with MODIS-derived LST. Mildrexler et al. (2011) originally noted the overestimation of LST in MODIS data. The current report describes specific regional and temporal aspects of the temperature uncertainty. Comparisons across a large area for an extended time period can minimise the error, i.e. geographical and temporal overestimation is balanced by underestimation. The greatest degree of overestimation occurs in the vicinity of the Mediterranean basin, where daytime overestimates exceed those collected during the night. Bonan (2008) proposed a mechanism explaining LST deviations as due to the ground vegetal cover. At high temperatures, and in non-forested cover types such as those typical of Mediterranean-type ecosystems, LST captures irradiative and thermodynamic features of Earth's surface more faithfully than Tair. Desert areas, shrub-lands, grasslands, savannas, and croplands have a maximum LST that is $5-10{ }^{\circ} \mathrm{C}$ hotter than the corresponding maximum Tair. Wan et al. (2004) originally recognised this bias in data from semi-arid and arid regions. In addition to the ecosystem effects on LST estimates, we identified pronounced other effects related to the time of satellite orbital transit. Transit times for both Terra and Aqua satellites over a given area do not always correspond with the time of maximum or minimum temperature on a given day.

The MODIS LST bias is not technically an error in raw data but rather a limitation of interpretation. This inconsistency in LST temperatures is therefore difficult to reconcile with post-hoc methods. Correcting MODIS data requires a dense network of ground recording stations to obtain accurate temperature estimates. This sort of operation (Hengl et al., 2011) can be implemented but only for studies of parasite habitats and life cycles over a relatively small area (Neteler, 2010; Neteler et al., 2011). The bias introduced by these uncertainties can affect interpretations of life cycle and development models of ticks and other arthropods. We analysed the propagation of temperature uncertainties in tick development models to quantify the bias. Temperature uncertainties range from $2-4 \%$ during the summer at southern latitudes. A 2-4\% overestimation in tick development introduced during successive 10-day intervals could lead to as much as $12 \%$ overestimation on the monthly scale. Ticks have four temperature-dependent development periods, i.e. oviposition, egg maturation, larva-nymph molting and nymph-adult molting (Randolph, 1998). These stages do not account for the periods of questing, which are not only modulated by temperature but also by humidity (Randolph, 1998). We did not reiterate the analysis for each developmental period, since the error has the same magnitude regardless of the equation. However, the propagation of the temperature error introduced into models by MODIS data may seriously obscure estimates of development, climate-influenced plant and animal life cycle events (phenology) or process-driven predictive mapping of the parasite.

Using flags issued by the MODIS scientific team to filter the dataset and remove non-valid pixels can serve to balance the overall error in LST measurements. These operations specifically balance outliers in the error distribution but regional-scale errors persist in the data. Although the absolute temperature error is higher in areas of the Mediterranean basin, underestimation of LST is potentially more problematic at northern latitudes, which are at risk for the spread of $I$. ricinus and their associated pathogens (Jaenson and Lindgren, 2011; Jaenson et al., 2012). The distribution of this organism is currently limited by low temperatures that characterise the higher latitudes (Jaenson and Lindgren, 2011) or high-altitude localities (Danielova et al., 2006) since low temperatures prevent tick maturation. Life cycle models for these latitudes can underestimate tick development rates by an average of $1 \%$ for every 10 -day period throughout the year. The most biased estimations could 
theoretically result in $36 \%$, uncertainties for the entire year. Satellite-derived temperature data could also indicate a northern limit of viable tick development that would not coincide with its actual range according to ground-based temperature measurements.

Our results indicate that MODIS LST data can provide potentially biased Tair estimates when entered into process-driven models of parasite development rates. Satellite measurements are typically used as a direct estimator of mean temperature within a given time-frame, without data filtering or other steps to correct for bias. Our results suggest that Terra (daytime) and Aqua (in the night) in combination provide adequate estimates of maximum or minimum temperatures for a given time period, but they do not provide reliable mean temperature estimates, which are commonly used in many process-driven developmental models. The use of minimum - maximum temperature bounds over a 10-day interval, with removal of invalid pixels provides adequate local estimates of Tair as well as reliable Tair estimates over wide-ranging areas.

While it is legitimate to use MODIS-derived LST data to generate parasite development models, technical errors as recognised by the MODIS team (Wan et al., 2004; Wan, 2008; Wan and Li, 2011) must be biologically interpreted before firm conclusions can be drawn. Estimations of developmental rates from MODISderived information should ideally only be compared with similar data developed with the same raw information. This precludes the direct comparison among models built on different raw variables such as, for example, those derived from diverse future climate scenarios based on interpolations of ground station data. Ticks develop and quest only on the first centimetres of the vegetation layer. Therefore, neither climate data obtained from ground recording climate stations nor satellite products are free of significant errors, thereby introducing a potential noise that should be considered (Estrada-Peña et al., 2012). In any case, every biological model should ideally be built on the variables that best describe the processes. No general rules for a "gold standard" are available because issues of spatial and temporal resolution operate at every level of the life cycle analysed (Randolph, 2002).

\section{Conclusion}

Data from this study suggest that satellite-sensed information can provide the basic background, against which models for development and mortality rates of health-threatening arthropods can be constructed. However, a thorough comparison with ground esti- mates of the given regional climate is necessary to provide the background of variability and the framework to improve the quality of the raw data.

\section{References}

Austin MP, 2002. Spatial prediction of species distribution: an interface between ecological theory and statistical modelling. Ecol Model 157, 101-118.

Bonan GB, 2008. Forests and climate change: forcings, feedbacks, and the climate benefits of forests. Science 320, 14441449.

Brooker RW, Britton AJ, Gimona A, Lennon JJ, Littlewood NA, 2011. Literature review: species translocations as a tool for biodiversity conservation during climate change. Scottish Natural Heritage Commissioned Report 440, Inverness, United Kingdom.

Danielova V, Rudenko N, Daniel M, Holubova J, Materna J, Golovchenko M, Schwarzova L, 2006. Extension of Ixodes ricinus ticks and agents of tick-borne diseases to mountain areas in the Czech Republic. Int J Med Microbiol 296, 48-53.

Dobson ADM, Finnie TJR, Randolph, SE, 2011. A modified matrix model to describe the seasonal population ecology of the European tick Ixodes ricinus. J Appl Ecol 48, 1017-1028.

Estrada-Peña A, 1999. Geostatistics and remote sensing using NOAA-AVHRR satellite imagery as predictive tools in tick distribution and habitat suitability estimations for Boophilus microplus (Acari: Ixodidae) in South America. National Oceanographic and Atmosphere Administration-Advanced Very High Resolution Radiometer. Vet Parasitol 81, 73-82.

Estrada-Peña A, Ayllón N, de la Fuente J, 2012. Impact of climate trends on tick-borne pathogen transmission. Front Physiol, 3, 64-79.

Estrada-Peña A, Venzal JM, 2007. Climate niches of tick species in the Mediterranean region: modeling of occurrence data, distributional constraints, and impact of climate change. J Med Entomol 44, 1130-1138

Guis H, Caminade C, Calvete C, Morse AP, Tran A, Baylis M, 2012. Modelling the effects of past and future climate on the risk of bluetongue emergence in Europe. J R Soc Interface 9, 339-350.

Guisan A, Zimmermann NE, 2000. Predictive habitat distribution models in ecology. Ecol Model 135, 147-186.

Guisan G, Thuiller W, 2005. Predicting species distribution: offering more than simple habitat models. Ecol Lett 8, 9931009.

Hancock PA, Brackley R, Palmer SCF, 2011. Modelling the effect of temperature variation on the seasonal dynamics of Ixodes ricinus populations. Int J Parasitol 41, 513-522.

Hay SI, Packer MJ, Rogers DJ, 1997. The impact of remote sensing on the study and control of invertebrate intermediate hosts and vectors for disease. Int J Remote Sens 18, 2899 . 
2930.

Hay SI, Tatem AJ, Graham AJ, Goetz SJ, Rogers DJ, 2006. Global environmental data for mapping infectious disease distribution. Adv Parasitol 62, 37-77.

Hengl T, Heuvelink GBM, Percec TM, Pebesma E, 2011. Spatio-temporal prediction of daily temperatures using timeseries of MODIS LST images. Theor Appl Climatol 107, 265 277.

Hijmans RJ, Cameron SE, Parra JL, Jones PG, Jarvis A, 2005. Very high resolution interpolated climate surfaces for global land areas. Int J Climatol 25, 1965-1978.

Jaenson TGT, Jaenson DGE, Eisen L, Petersoon E, Lindgren E, 2012. Changes in the geographical distribution and abundance of the tick Ixodes ricinus during the past 30 years in Sweden. Parasit Vectors 5, 8.

Jaenson TG, Lindgren E, 2011. The range of Ixodes ricinus and the risk of contracting Lyme borreliosis will increase northwards when the vegetation period becomes longer. Ticks Tick Borne Dis 2, 44-49.

Jin M, Dickinson RE, 2010. Land surface skin temperature climatology: benefitting from the strengths of satellite observations. Environ Res Lett 5, 1-13.

Justice CO, Townshend JRG, Vermote EF, Masuoka E, Wolfe RE, Saleous N, Roy DP, Morisette JT, 2002. An overview of MODIS land data processing and product status. Remote Sens Environ 83, 3-15.

Liang S, Liu R, Liu J, 2006. Estimation of systematic errors of modis thermal infrared bands. Institute of Electronical and Electronics Engineers.

Mildrexler DJ, Zhao M, Running SW, 2011. A global comparison between station air temperatures and MODIS land surface temperatures reveals the cooling role of forests. J Geophys Res $116,15$.

Munderloh UG, Kurtti TJ, 2010. Emerging and re-emerging tick-borne diseases: new challenges at the interface of human and animal health. Workshop "Critical needs and gaps in understanding prevention, amelioration, and resolution of lyme and other tick-borne diseases: the short-term and longterm outcomes" Washington, DC, October 11-12, 2010.

Neteler M, 2010. Estimating daily land surface temperatures in mountainous environments by reconstructed MODIS LST data. Remote Sens 2, 333-351.

Neteler M, Roiz D, Rocchini D, Castellani C, Rizzoli A, 2011. Terra and Aqua satellites track tiger mosquito invasion: modeling the potential distribution of Aedes albopictus in northeastern Italy. Int J Health Geograph 10, 49.

Ogden NH, Barker IK, Beauchamp G, Brazeau S, Charron DF, 2006. Investigation of ground level and remote-sensed data for habitat classification and prediction of survival of Ixodes scapularis ticks in habitats of southeastern Canada. J Med
Entomol 43, 403-414.

Ogden NH, Maarouf A, Barker IK, Bigras-Poulin M, Lindsay LR, Morshed MG, O'Callaghan CJ, Ramay F, Waltner-Toews D, Charron DF, 2005. Climate change and the potential for range expansion of the Lyme disease vector Ixodes scapularis in Canada. Int J Parasitol 36, 63-70.

Ostfeld RS, Glass GE, Keesing F, 2005. Spatial epidemiology: an emerging (or re-emerging) discipline. Trends Ecol Evol 20, 328-336.

Patz JA, Campbell-Lendrum D, Holloway T, Foley JA, 2005. Impact of regional climate change on human health. Nature 438, 310-317.

Randolph S, 2002. Quantitative ecology of ticks as a basis for transmission models of tick-borne pathogens. Vector-Borne Zoonot 2, 209-215.

Randolph SE, 1998. Ticks are not insects: consequences of contrasting vector biology for transmission potential. Parasitol Today 14, 186-192.

Randolph SE, 2004. Tick ecology: processes and patterns behind the epidemiological risk posed by ixodid ticks as vectors. Parasitology 129, 37-65.

Randolph SE, Green RM, Hoodless AN, Peacey MF, 2002. An empirical quantitative framework for the seasonal population dynamics of the tick Ixodes ricinus. Int J Parasitol 32, 979989.

Rogers DJ, Randolph SE, 2006. Climate change and vectorborne diseases. Adv Parasitol 62, 345-381.

Rogers DJ, Randolph SE, Snow RW, Hay SI, 2002. Satellite imagery in the study and forecast of malaria. Nature 415, 710715 .

Scharlemann JPW, Benz D, Hay SI, Purse BV, Tatem AJ, 2008. Global data for ecology and epidemiology: a novel algorithm for temporal fourier processing MODIS data. PLoS One 3, e1408.

Tatem AJ, Hay SI, Rogers DJ, 2006. Global traffic and disease vector dispersal. Proc Natl Acad Sci USA, 6242-6247.

Townshend JRG, Justice CO, 2002. Towards operational monitoring of terrestrial systems by moderate-resolution remote sensing. Remote Sens Environ 83, 351-359.

Wan Z, 2008. New refinements and validation of the MODIS land-surface temperature/emissivity products. Remote Sens Environ 112, 59-74.

Wan Z, Li ZL, 2011. MODIS land surface temperature and emissivity. In: Remote sensing and digital image processing vol. 11, Land Remote Sensing and Global Environmental Change. B. Ramachandran B, Justice CO, Abrams MJ (eds). New York: Springer, 563-577 pp.

Wan Z, Zhang Y, Zhang Q, Li ZL, 2004. Quality assessment and validation of the MODIS global land surface temperature. Int J Remote Sens 25, 261-274. 